\title{
EMERGENCY SURGICAL TREATMENT FOR MALIGNANT BOWEL OBSTRUCTION
}

\author{
R. Madjov, P. Arnaudov, K. Georgiev, V. Bojkov \\ University Hospital “St. MARINA”, Medical University - Varna, Bulgaria
}

Reviewed by: Assoc. prof. K. Ivanov, PhD

\section{SUMMARY}

Bowel obstructions are one of the most frequent reasons for emergency surgery in advanced staged cancer patients. The aim of the study was to review the presentation, treatment procedures and outcomes of malignant bowel obstructions and to identify the risk factors related with poor prognosis. 376 patients were studied. Data included age, sex, medical history, presenting symptoms, physical findings on admission, ASA class, operative procedures, postoperative morbidity and mortality. The most common complaints were abdominal pain, vomiting, nausea and constipation. Distension, altered bowel sound, pain and tenderness were most common findings. Greatest value for exact diagnosis had US, X-ray examination, endoscopic examination. Primary neoplasm of the bowel was found in $84 \%$. Obstruction due to recurrence/metastasis - in $16 \% .87 \%$ were with large bowel obstruction; ileum - in 3\%; both ileum and colon were involved in $8 \%$. Perforation found in $14 \%$. Curative resection was performed in $74,8 \%$. Extended bowel resection - in $24 \%$.Palliative procedures performed in patients with distant metastasis or locally advanced cancer $-\mathbf{2 5 , 2 \%}$. Overall morbidity $-26,9 \%$ and mortality $-11,6 \%$; but patients with serious coexisting disease had significantly higher incidence of postoperative death $-33 \%$.

Keywords: Colorectal cancer, Malignant bowel obstruction, Emergency surgery, Endstage cancer patients

\section{INTRODUCTION}

Bowel obstructions (with or without perforations) are one of the most frequent reasons for emergency abdominal surgery in advanced cancer patients.

Malignant bowel obstructions are still a challenging problem for: gastroenterologists, endoscopists and surgeons, and they carry high morbidity rate and high mortality rate. The incidence of bowel obstructions according to the literature ranges from:

- $25-35 \%$ in pts with advanced colorectal cancer

- 5 - 32 in pts with advanced ovarian cancer

- $2-5 \%$ in pts with huge retroperitoneal tumors

- $1-3 \%$ in pts with advanced gastric cancer

Large bowel obstruction is caused mainly from:

- malignancy;

- volvulus;

- diverticular stricture

and these entities account for approximately $90 \%$ of all causes of mechanical obstruction of the colon. Over $50 \%$ of colonic obstructions are malignant in nature and primary colorectal cancer being the predominant cause. Less common causes of colonic obstruction include:

Address for correspondence:

Rossen Madjov, $2^{\text {nd }}$ Department of Surgery, University Hospital

"St. Marina", ${ }^{\text {st }}$ Nr. Smirnensky blvd, Varna - 9010, BULGARIA

Email:madjov@mu-varna.bg
- Inflammatory bowel disease;

- Radiation stricture;

- Fecal impaction;

- Intussusception

- Ischemic stricture

\section{THE AIM}

- to review the clinical presentation;

- treatment procedures and outcomes of malignant bowel obstructions

- to identify the risk factors related with poor prognosis

\section{PATIENTS AND RESULTS}

Original hospital records of 376 patients admitted in the $2^{\text {nd }}$ Department of abdominal surgery with malignant bowel obstruction were reviewed. Their mean age was 64,6 years (varied between $23-97$ years).

Primary neoplasm of the bowel was found in 315 (83,7\%) of the patients. Other reasons diagnosed in our series were:

- recurrence - in 13,6\% of our cases /

- metastases

- ovarian cancer

- retroperitoneal tumor 
According to the site of the bowel obstruction the distribution of our patients was as follows:

- large bowel obstruction $\quad-87 \%$

- obstruction involving ileum

$-3 \%$

- both ileum and colon involved

$-8 \%$

- obstruction confined to gastric outlet

$-2 \%$

Perforation associated with obstruction was found in 64 $(17,0 \%)$ of our patients $(67 \%$ at the site of obstructive neoplasm). Peritonitis was diagnosed pre- and intra-operatively in 59 patients.

The distribution of the patients according to the duration of the clinical signs and symptoms was:

- $<3$ days $\quad-54,5 \%$

- 4-6 days $\quad-24,8 \%$

- $>7$ days $\quad-20,7 \%$

The most common presenting complaints of the patients at the time of their admission in the hospital were: abdominal pain, vomiting, nausea, constipation.

The most common findings on physical examination of our patients were: Distension, Abdominal pain, Tenderness in palpation, Altered bowel sounds

Severe coexisting diseases were diagnosed in $61 \%$ of the patients (most of them were advanced aged). Most common were:

- Congestive heart failure

- Chronic obstructive pulmonary disease

- Diabetes mellitus

Most valuable for the exact preoperative diagnosis in our patients were:

- careful examination of the patient starting in the emergency room

- plain abdominal X-ray films

- preoperative Ultrasonography

- endoscopic examinations + biopsies (R R S, F S S, F C S)

- C T scans / less common/

Tabl. 1. - Comparison between right-sided and left-side colon tumors

\begin{tabular}{||l|c|c|c||}
\hline \multicolumn{1}{|c|}{ Characteristic } & $\begin{array}{c}\text { Right-side } \\
(25 \mathrm{pts})\end{array}$ & $\begin{array}{c}\text { Left side } \\
(78 \mathrm{pts})\end{array}$ & P value \\
\hline male / female & $13 / 12$ & $43 / 35$ & 0,48 \\
\hline Severe coexisting disease & $7(28 \%)$ & $27(35 \%)$ & 0,36 \\
\hline Complete obstruction & $10(40 \%)$ & $30(39 \%)$ & 0,53 \\
\hline Perforation & $6(24 \%)$ & $9(12 \%)$ & 0,19 \\
\hline ASA II & - & $4(5 \%)$ & \\
\hline ASA III & $10(40 \%)$ & $32(41 \%)$ & 0,59 \\
\hline ASA IV & $13(52 \%)$ & $33(42 \%)$ & \\
\hline ASA V & $2(8 \%)$ & $9(12 \%)$ & \\
\hline Resection & $24(96 \%)$ & $65(83 \%)$ & 0,18 \\
\hline Curative resection & $20(80 \%)$ & $54(74 \%)$ & 0,53 \\
\hline Severe postop. morbidity & $2(8 \%)$ & $20(26 \%)$ & $0,04 *$ \\
\hline Mortality & $1(4 \%)$ & $16(21 \%)$ & $0,04 *$ \\
\hline \hline
\end{tabular}

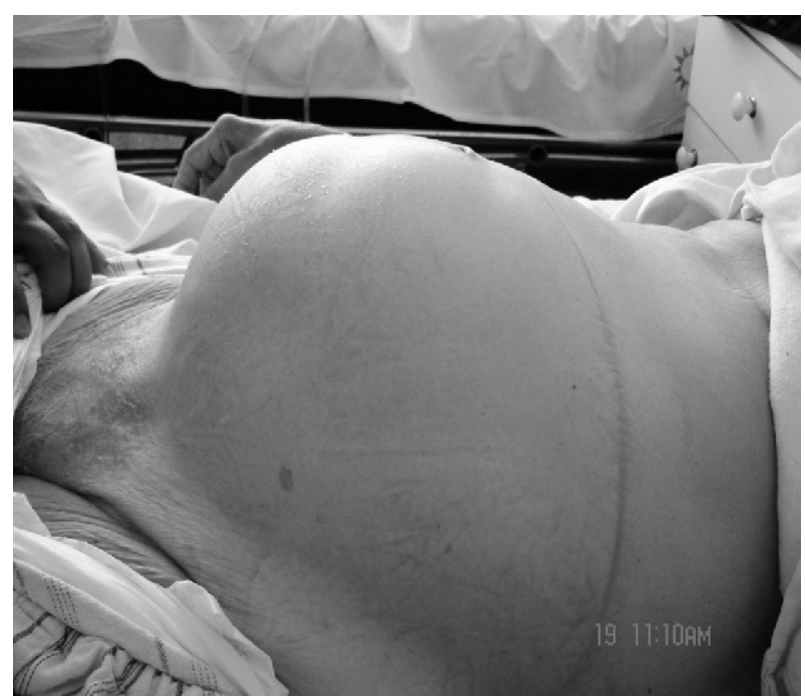

Fig. 1. 6th day malignant colonic obstruction

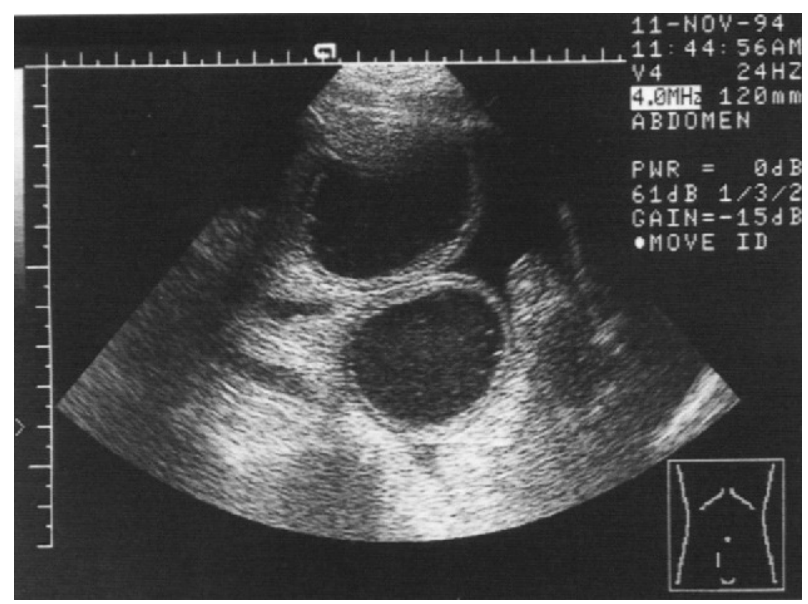

Fig. 2. US in patient with malignant bowel obstruction

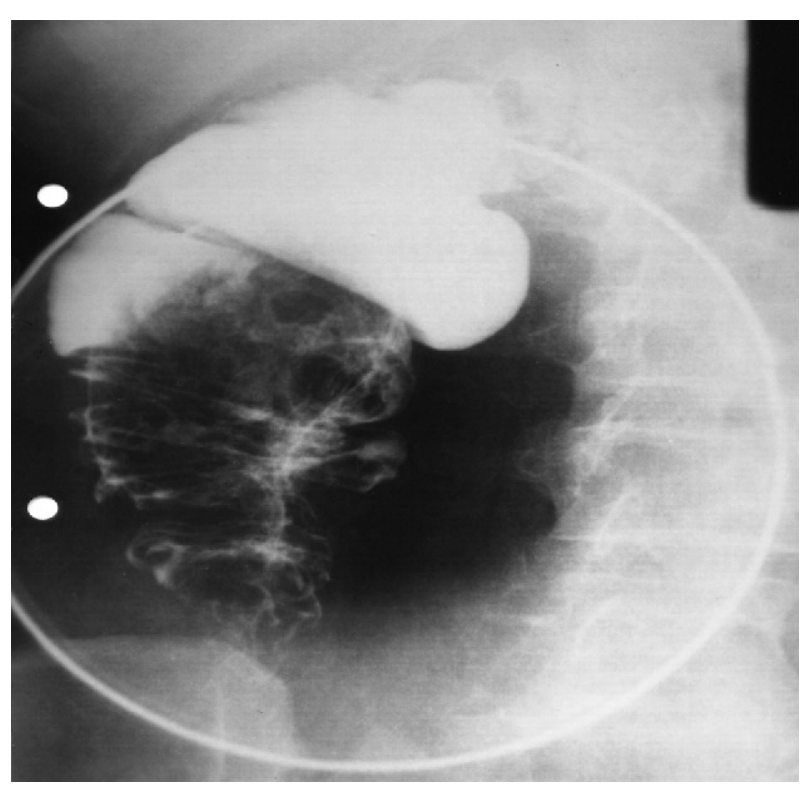

Fig. 3. Intussusception caused by Carcinoma coli 


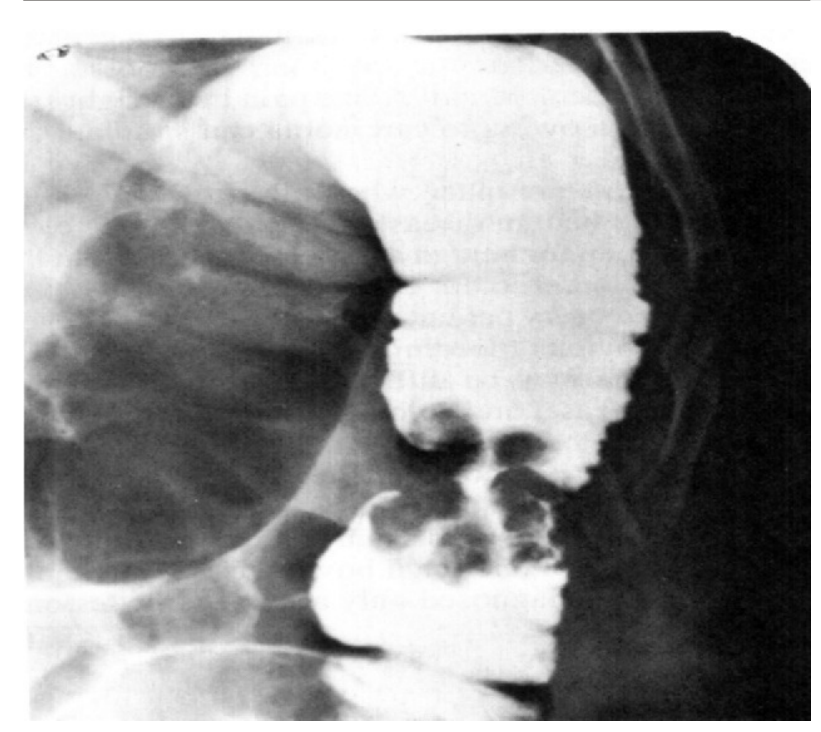

Fig. 4. Contrast Ro-graphy - Carcinoma coli

All of the patients underwent operative procedures - urgent operation up to the first 24 hours were performed in 344 patients. 32 of the patients were treated conservatively initially, followed by elective operative procedures in 24 of them.

Curative resection was performed in $262(76,1 \%)$ of the patients. Extended bowel resection - performed in 82 $(23,8 \%)$ of the patients.

Tabl 2. - Correlation of preoperative performance status with morbidity and mortality rate

\begin{tabular}{|l|c|c|c||}
\hline \multicolumn{1}{|c|}{ Status } & No of Pts & Morbidity & Mortality \\
\hline ASA II & 49 & 1 & None \\
\hline ASA III & 48 & $8(17 \%)$ & $3(6 \%)$ \\
\hline ASA IV & 59 & $20(34 \%)$ & $16(27 \%)$ \\
\hline ASA V & 14 & $9(64 \%)$ & $7(50 \%)$ \\
\hline \hline
\end{tabular}

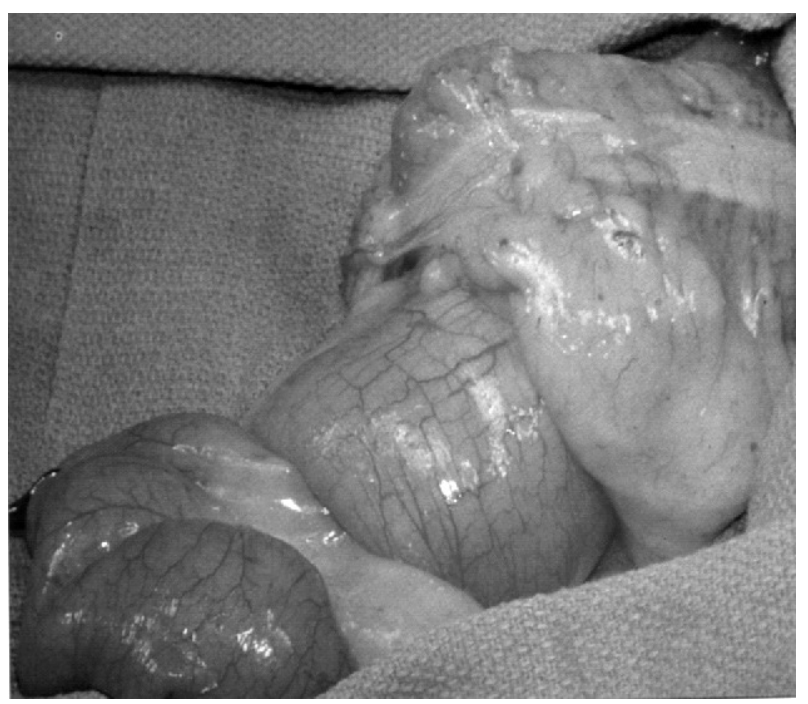

Fig. 5. Ileo-coeco-colonic intussusception

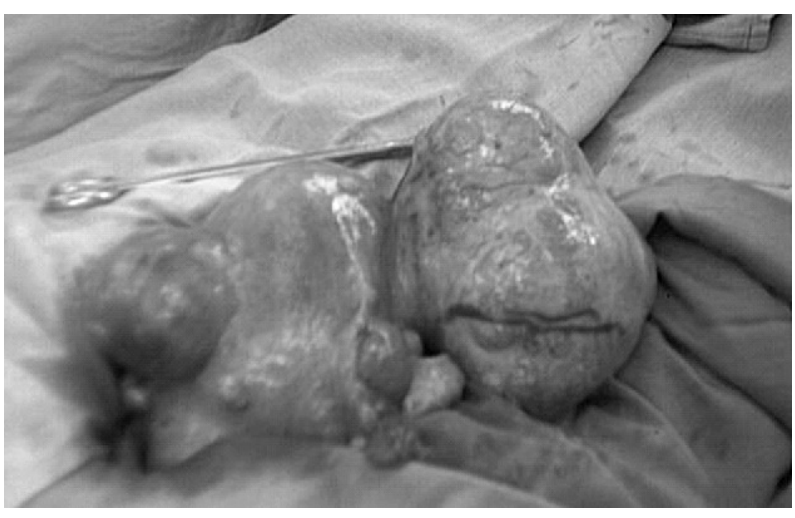

Fog. 6. Ovarian cancer causing small bowel obstruction

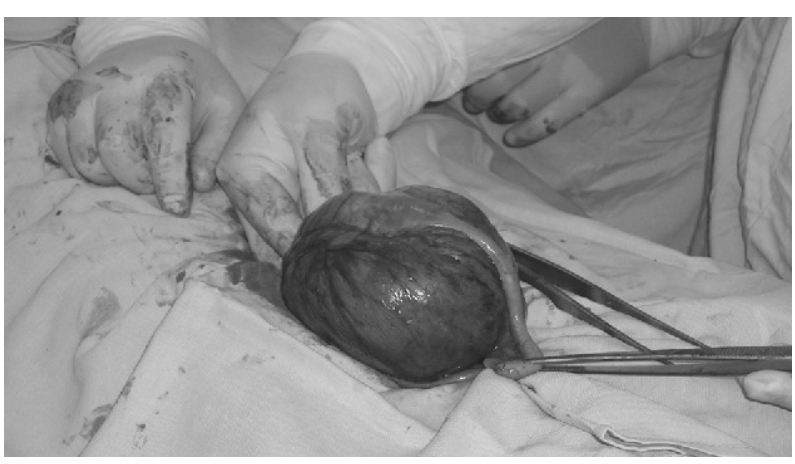

Fig. 7. Leyomyoma coeci

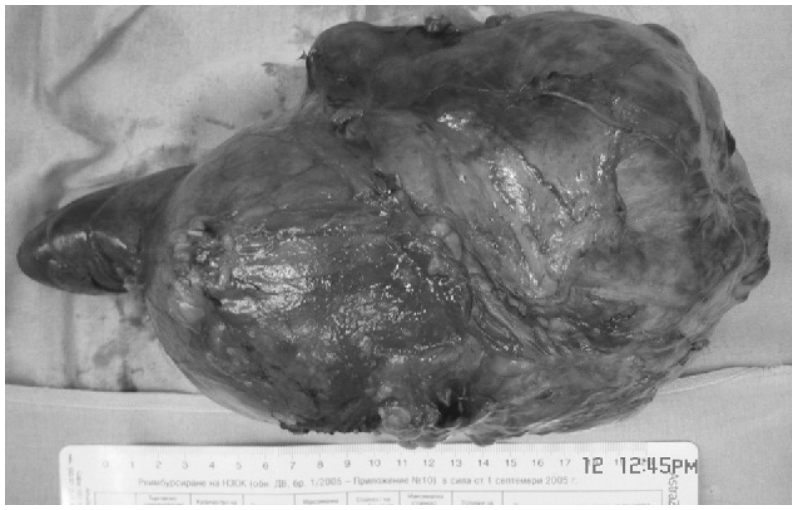

Fig. 8. Retroperitoneal tumor causing intestinal obstruction

The main controversy in the management of obstructing colon cancers is the operative approach to those in the left and sigmoid colon.

Historically a three-stage procedure was performed:

1/ Divert; 2/ Resect; 3/ Reverse stoma

This was superseded by a two-stage procedure such as Hartmann's operation (resect without anastomosis; restore continuity later).

More recently the concept of resection, on-table lavage to empty the resected colon of stool and primary re-anastomo- 


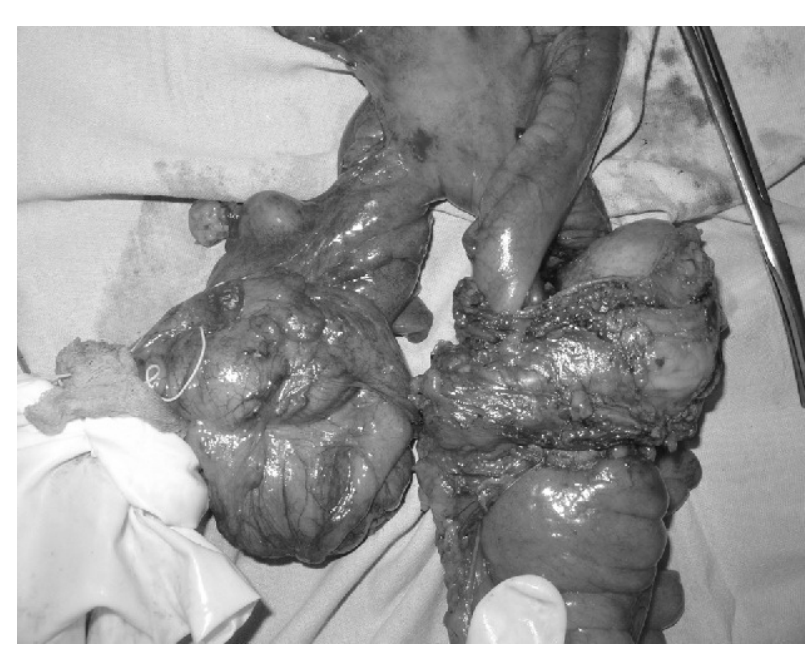

Fig. 9. A. J. 68 years $/ 2005 /$ - Ca coeci \& Ca sigmae

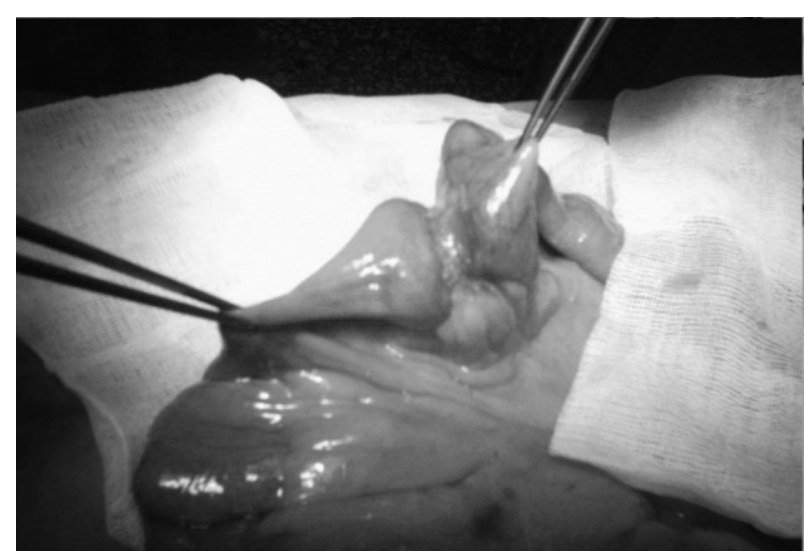

Fig. 10. Obstruction caused by carcinoma intestine tenuae

sis has been proposed to avoid a stoma and second operation.

Similarly subtotal colectomy with primary anastomosis of the terminal ileum to the colon at the distal resection margin has been suggested as a one-stage approach - this procedure may be necessary if the obstruction has caused serosal tears or ischemic necrosis in the coecum.

Palliative procedures were performed in $23,9 \%$ of the patients. Palliation included:

- tumor resection

- decompressive stoma /ileo-; coeco- transverse- or sigmostomy/

Palliation usually was done in patients with distant metastases or locally advanced cancer.

Postoperative complications were diagnosed in $26,9 \%$ of our patients. Postoperative mortality was 5,6\%. But patients with serious coexisting disease had significantly higher incidence of postoperative complications (41\%) and postoperative death (33\%).

\section{CONCLUSIONS}

- Primary neoplasms are usually removable in cases of malignant bowel obstructions.

- Emergency surgical management with curative intention requires careful assessment.

- Advanced age, complete obstruction, coexisting diseases and requirement for extended bowel resections are not contraindications for emergency surgical treatment aimed to relieve the symptoms.

- Emergency indication for surgical treatment of intra-abdominal cancer complications were established as clinical signs of acute abdomen with intestinal obstruction.

- All patients should undergo fluid resuscitation for correction of the electrolyte disturbances and optimization of the performance status before operative procedure.

- Mostly the location of the neoplasm and general condition of the patient determines the surgical strategy.

- Primary resection and anastomosis should be considered in the presence of well prepared bowel and absence of generalized peritonitis, good performance status of the patient and acceptable morbidity and mortality risk.

- $60 \%$ of the patients with CRC present with stage II or III of the disease, which will recur with metastatic or locally invasive disease in approximately $35-40 \%$ of them.

- Surgery is the cornerstone of therapy for such tumors and 70 to $80 \%$ of patients with such tumors can be resected with curative intent. As a result, hundreds of thousands of people with resected CRC are candidates for surveillance.

- Among patients who have undergone resection for localized disease, the Five-years survival rate is $90 \%$. The rate decreases to $65 \%$ when metastases to regional lymph node are present. More than $90 \%$ of relapses occur by 5 years (the majority within 3 years)

- The most common sites of recurrence are: Liver, Local site, Abdomen, Lung

- Palliative interventions should be reserved for relieving the symptoms in the patients with poor general condition.

- Ethical principles related to medical and surgical treatment in patients with advanced CRC can be considered under the following headings:

- eneficence /do good/

- Non maleficence /do not harm/

- Autonomy /respect the rights of the individual patient/

- Factors other than disease stage that may impair survival in colorectal cancer are;

- Perforation

- Obstruction

- Poor differentiation

- Mucinous/signet ring histology 
- Lympho-vascular invasion

- Perineural invasion

- DNA aneuploidy

- Elevation of CEA

\section{REFERENCES}

1. Alan MY Surgical emergencies in the cancer patient. In Norton JA, Cang AE, Stephen FL et al. (Eds) Surgery. New York; Springler, 2000; 1823-1837

2. Bold RJ Oncologic emergencies. In Feig BW, Berger, Fuhrman GM (Eds) The M.D. Anderson surgical oncology handbook. Philadelphia; Lippincott Williams \& Williams 1999; 425-446

3. Douglas JS - Surgical emergencies. In Devita VT, Hellman S, Rosenborg SA (Eds) Cancer Principles and Practice Oncology. $5^{\text {th }}$ ed. Philadelphia; Lippincott-Raven, 1997; 2500-2522
4. Feuer DJ, Broadley KE, Shepherd JH, Bartron D. Systematic review of surgery in malignant bowel obstruction in advanced gynecological and gastrointestinal cancer. Gynecol. Oncol. 1999; vol. 75:313-322

5. Gallick HL, Weaver DW, Sachs RJ et al. Intestinal obstruction in cancer patients: an assessment of risk factors and outcome. Am Surg. 1986; vol. 52:434-437

6. Kyllonen LEJ Obstruction and perforation complicating colorectal carcinoma. Acta Chir Scand 1987; vol. 153:607-610

7. Ripamonti C, Twycross R, Baines M, et al. Clinical practice recommendations for the management of bowel obstruction in patients with end stage cancer. Support Care Cancer 2001; 9:223-233

8. Woolfson RG, Jennings K, Whalen GF - Management of bowel obstruction in patients with abdominal cancer. Arch Syrg 1997; vol. 132:1093-1097 